\title{
Editorial of the Special Issue: "Artificial materials for advanced applications in electromagnetics and mechanics"
}

\author{
Christos Argyropoulos ${ }^{1}$ and Alessio Monti ${ }^{2, *}$ \\ 1 University of Nebraska-Lincoln, 1400 R St, Lincoln, NE 68588, USA \\ 2 Niccolò Cusano University, Via Don Carlo Gnocchi 3, 00166 Roma, Italie
}

Received 24 March 2017 / Accepted 29 April 2017

In the past 10 years, metamaterials have been established at the forefront of research in several diverse areas, such as electromagnetics, acoustics, and mechanics. The interdisciplinary nature of metamaterial research is the key behind its currently successful status.

This editorial article introduces the second edition of the Special Issue of the Metamaterials Congress born from a partnership between the "International Congress on Advanced Electromagnetic Materials in Microwaves and Optics Materials" and the "EPJ Applied Metamaterials" (EPJ AM) Journal. The last edition of the Metamaterials Congress has been hosted by the Foundation for Research and Technology - Hellas (FORTH) in the beautiful island of Crete, Greece. As usual, the Congress comprised a 4-day Conference (19-22 September) and a 2-day Doctoral School (17-18 September) and more than 280 oral and poster presentations presented by delegates representing over 40 countries. The Congress also included some special events dedicated to the 10th anniversary of the series that has started in 2007 in Rome, Italy and, since then, has established itself as one of the most active conference in the field of artificial engineered materials or metamaterials.

We are particularly glad to serve as Guest-Editors for this second edition of the Special Issue that contains a representative collection of the topics debated during the Conference. This Special Issue covers both state-of-the-art developments and new research topics in the fundamentals and applications of metamaterials in different scientific and technical fields.

The paper by S. G. Rodrigo is focused on the design of a sensing system for the detection of spectral signatures of gases at the terahertz regime. The system consists in an initially opaque holey metal film where the introduction of a gas provokes the appearance of spectral features in transmission and reflection. The mechanism behind this effect is due to the phenomenon of absorption-induced transparency. A practical example of the proposed terahertz gas sensor to detect tiny amounts of hydrocyanic acid is demonstrated.

\footnotetext{
*e-mail: alessio.monti@unicusano.it
}

The paper by A. Monti et al. presents a new mantle cloaking approach to design Yagi-Uda antennas with low scattering profile. The authors present a general design procedure of such cloaked antennas, considering that both antenna and cloak requirements must be simultaneously fulfilled. Then, they prove that a cloaked Yagi-Uda antenna can be installed in close proximity to other radiators without affecting their performance. The proposed antenna systems may have a strong impact in the design of compact overcrowded platforms for both terrestrial and satellite applications.

The paper by P. A. Huidobro et al. demonstrates a new tunable plasmonic metasurface to achieve perfect absorption at terahertz frequencies. The presented metasurface design is based on a graphene monolayer subject to a spatially periodic gate bias, which, thank to surface plasmons in the graphene, acts as a tunable and extremely compact absorber for terahertz radiation. The proposed structure can be used as a modulator, and its frequency of operation can be changed by scaling its size or adjusting the doping level. It opens up the possibility of ultra-compact photonic components with reconfigurable applications.

The paper by $\mathrm{C}$. Valagiannopoulos et al. introduces a new class of materials, named Conjugate Matched Layers, whose properties derive from a combination of Perfectly Matched Layers and Double-Negative media. In particular, these materials achieve conjugate matching with free-space for every possible mode, including the evanescent ones. Moreover, the effect of a single particle placed in their close proximity is evaluated and, remarkably, it is found that such a material allows overcoming the black-body absorption limit.

In the paper by N.L. Tsitsas, an efficient integral equation modeling of scattering by a gradient dielectric metasurface is investigated. The proposed technique is based on the method of moments with entire domain basis functions and is characterized by high numerical stability and efficiency and controllable accuracy. Moreover, it is shown that an appropriate selection process allows designing reflection filters in the microwave regime and generating anomalous reflection 
and transmission effects from a metasurface composed by conventional materials.

The paper by Y. Urade et al. proposes a theoretical study about the possibility to dynamically switch the handedness of a planar chiral checkerboard-like metasurface by modulating the local surface impedance of the structure. In particular, it is shown that the polarity of the planar chirality of the metasurface can be inverted by switching the sheet impedance at the interconnection points of the checkboard-like metasurface. The expected behavior is explained from both a microscopic and a macroscopic point of view and is confirmed by numerical simulations.

Finally, the paper by K.V. Sreekanth et al. focuses on the use of hyperbolic metamaterials for the design of plasmonic biosensors for fluid biopsy with single molecule sensitivity. The biosensor platform is composed by a combination of a metallic diffraction grating and a hyperbolic metamaterial and allows achieving an extreme sensitivity close to a single molecule level. The paper also contains an experimental realization of the sensor and several measured results.

To conclude, we would like to acknowledge the many people that have made possible the publication of this Special
Issue. First of all, our sincere thanks go to the EPJ AM Editorin-Chief, Prof. Yang Hao, and to the Congress General Chairs, Profs. Filiberto Bilotti and Andrea Alù, for having established a partnership between the EPJ AM journal and the Metamaterials Congress and for giving us the possibility to edit the first two editions of this Special Issue. A special acknowledge goes also to the EPJ AM Editorial Staff whose promptly and accurate help allowed the timely publication of all the papers. The Guest Editors sincerely thank the authors for having submitted the exciting results of their research to this Special Issue. We are also very grateful to the reviewers who dedicated their time and expertise to increase the scientific quality of the submissions. Last but not least, we sincerely thank all the readers interested in the papers published on this Special Issue. We hope you may find here new exciting ideas for your research activities.

Cite this article as: Argyropoulos C \& Monti A: Editorial of the Special Issue: "Artificial materials for advanced applications in electromagnetics and mechanics". EPJ Appl. Metamat. 2017, 4, E1. 


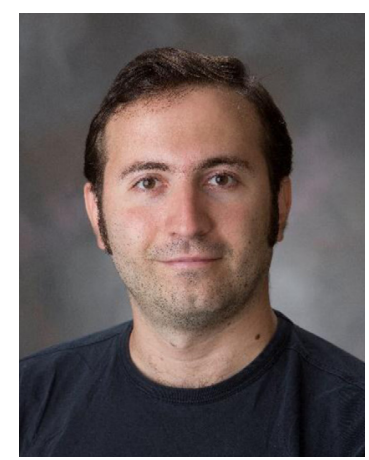

Christos Argyropoulos received the Diploma of Electrical and Computer Engineering from the Aristotle University of Thessaloniki, Greece (2006). He holds a M.Sc. degree in Communication Engineering from the Microwaves and Communication Systems group of the University of Manchester, UK (2007) and a Ph.D. degree in Electronic Engineering from the Antennas and Electromagnetics Group of the Queen Mary, University of London, UK (2011). After completion of his $\mathrm{PhD}$ studies, he accepted a Postdoctoral Fellowship position in the University of Texas at Austin, USA. Next (2013), he worked as a Postdoctoral Associate in the Center for Metamaterials and Integrated Plasmonics at Pratt School of Engineering, Duke University, USA. From September 2014, he is an Assistant Professor at University of Nebraska-Lincoln, Department of Electrical Engineering, where he established the metamaterials and integrated nanophotonics lab.

He has published over 140 technical papers in highly ranked journals and refereed conference proceedings, including five book chapters. His main research interests include linear and nonlinear plasmonics, active metamaterials, computational electromagnetics, numerical and analytical modeling of metamaterials and their applications, novel antenna design, transformation optics, thermal emission from plasmonic structures, graphene nanophotonics, new energy harvesting devices and acoustic metamaterials. He has received several travel and research awards, such as 2017 ONR summer faculty fellowship, Junior Researcher Award of the 2013 Raj Mittra Travel Grant, EPSRC Research Scholarship, Royal Academy of Engineering international travel grant and twice the Marie Curie Actions Grant to attend the European School of Antennas. He has given several invited talks and seminars to different conferences and universities. He served as Student Paper Competition co-chair at IEEE APS 2016 and as guest co-editor at the EPJ Applied Metamaterials special issue of the Metamaterials' Congress. He is a technical program committee member and special session on commercialization of metamaterials organizer at Metamaterials 2017 conference. He is Associate Editor at Optics Express and member of the Optical Society of America Traveling Lecturer program. He is treasurer at 16th Annual IEEE International Conference on Electro Information Technology. He is a senior member of IEEE, a full member of URSI Commission B, and member of IEEE Antennas and Propagation Society, IEEE Photonics Society, Optical Society of America, SPIE, American Physical Society and the Technical Chamber of Greece.

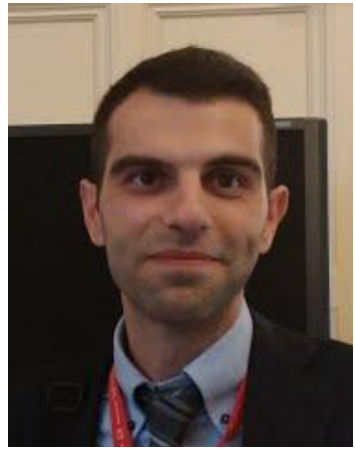

Alessio Monti was born in Rome, Italy, on February, 1987. He received the B.S. degree (summa cum laude) and the M.S. degree (summa cum laude) in electronic and ICT engineering both from Roma Tre University, Rome, in 2008 and 2010, respectively. From 2011 to 2013, he attended the Doctoral school in biomedical electronics, electromagnetics and telecommunications engineering at Roma Tre University in the research group led by Profs. Filiberto Bilotti and Alessandro Toscano. Currently, he is an assistant professor at Niccolò Cusano University, Rome, Italy.

Dr. Monti is member of the secretarial office of the International Association "Metamorphose-VI", and of the Editorial Board of the journal "EPJ Applied Metamaterials". In 2017, he has been appointed as Chairman of the Steering Commitee of the 11th International Congress on Engineered Material Platforms for Novel Wave Phenomena - Metamaterials' 2017. He is also member of the Technical Program Committee (TPC) of the IEEE International Symposium on Antennas and Propagation since 2016 and he has been member of the TPC of the International Congress on Advanced Electromagnetic Materials in Microwaves and Optics in the years 2014-2016. He has also been serving as a Technical Reviewer of the many high-level international journals related to electromagnetic field theory, metamaterials and plasmonics.

His research interests include the design and the applications of the microwave and optical engineered materials and metasurfaces and the design of cloaking devices for scattering cancellation at microwave and optical frequencies with a particular emphasis on their applications to the antenna theory.

https://sites.google.com/site/montialessio/ 